\title{
physiotherapie
}

\section{Der rote Fleck}

\author{
RED FLAGS ERKENNEN Nach einem Umzug hat Sabine Loose
} starke Schmerzen in beiden Ellenbogengelenken, auch der Nacken tut ihr weh. Die bei der Untersuchung gefundenen Befunde passen für Physiotherapeut Prof. Dr. Harry von Piekartz allerdings nicht so recht zusammen. Merkwürdig findet er auch etwas anderes: den kleinen roten Fleck auf Sabines Rücken.

S abine Loose, die in Wirklichkeit anders heißt, ist 23 Jahre alt, ledig und wohnt alleine. Sie hat eine Vollzeitstelle als Kassiererin und geht in ihrer Freizeit häufig mit ihrem Hund spazieren. Seit sie vor drei Wochen umgezogen ist, hat sie zunehmende dumpfe, tief sitzende Schmerzen in beiden Ellenbogengelenken, die unabhängig von bestimmten Bewegungen sind, sowie bilaterale Nackenschmerzen. Kopfschmerzen hat sie keine (๑ Abb. 1).

Durch die Einnahme von 50mg Paracetamol kann sie ihre Schmerzen (7/10) kurzfristig um zwei Punkte senken, doch nach ein paar Stunden ist der Schmerz wieder da. Obwohl Sabine Loose nicht mehr arbeitet als sonst, werden die Schmerzen zunehmend schlimmer.

Zusätzlich leidet sie seit vier Jahren unter Rückenschmerzen. Normalerweise sind diese diffus im Bereich der Lendenwirbelsäule (L4/L5) und werden zum einen stärker, wenn sie viel heben und tragen muss, zum anderen zwei bis drei Tage vor ihrer Menstruation. Seit sieben Tagen sind die Schmerzen eher "stechend“, und $\mathrm{ab}$ und zu hat Sabine auf der linken Seite ein brennendes Gefühl auf Höhe L4/L5. Zudem hat sie den Eindruck, dass die Beschwerden auf der linken Seite in den lateralen und zum Teil in den ventralen Oberschenkel ausstrahlen. Die gleiche Symptomatik spürt sie auf der rechten Seite - allerdings deutlich weniger. Wenn sie über ihre Haut am linken Oberschenkel streicht, fühlt es sich oft „pelzig“ an. Während der vergangenen Wochen hat sich die Stelle der Symptome auf ihren Oberschenkeln von medial nach lateral verlagert.

Ansonsten fühlt sich Sabine gesundheitlich nicht eingeschränkt. Sie ist lediglich häufig müde, seit sie in ihre neue Wohnung gezogen ist. Wenn sie schläft, erholt sie sich jedoch gut.

Was Sabine am meisten Sorgen bereitet, sind die Schmerzen in beiden Ellenbogengelenken. Morgens wacht sie mit diesen auf (VAS 3/10) und im Laufe des Tages werden sie immer schlimmer
(VAS5/10). Am Wochenende sind die Schmerzen weniger, aber immer noch vorhanden. Weil Sabine sich nicht erklären kann, warum die Beschwerden in der letzten Zeit schlimmer geworden sind, beschloss sie, ihren Hausarzt aufzusuchen, welcher sie zur Physiotherapie geschickt hat.

Die dumpfen, bilateralen Schmerzen in den Ellenbogengelenken hängen möglicherweise mit Sabines Nackenschmerzen oder mit neurodynamischen Dysfunktionen zusammen. Die Topografie der Schmerzen scheint allerdings nicht typisch neurodynamisch zu sein. Durch den Umzug könnte es möglicherweise zu einer Überlastung ihrer Arme gekommen sein. Das erklärt allerdings nicht, warum die Schmerzen in den Ellenbogen und im Nacken immer noch vorhanden und die stechenden und brennenden Rückenschmerzen zur gleichen Zeit entstanden sind. Sabine gibt keine Traumata des Nackens oder der Ellenbogen an und klagt auch nicht über Schmerzen oder Blockierungen in den Ellenbogengelenken, die auf eine intraartikuläre Dysfunktion oder eine "Gelenkmaus" hinweisen könnten - zumal eine solche Dysfunktion wahrscheinlich nicht auf beiden Seiten gleichzeitig auftreten würde. Hintergrund der plötzlichen multilokalen Beschwerden könnte eine beginnende System- oder neurologische Erkrankung sein. Daher frage ich als nächstes mögliche Hinweise auf neurologische Erkrankungen ab.

\section{Gezielte Fragen zu neurologischen Erkrankungen}

> Hatten Sie vorher schon einmal Sensibilitätsveränderungen in den Beinen oder Armen?

> Hat sich Ihre Kraft in den Armen und Händen bzw. in den Beinen und Füßen im Vergleich zu früher verändert?

$>$ Hatten Sie schon einmal plötzliche Krämpfe in den Armen oder Beinen?

> Bereiten Ihnen schnelle Hand- und Fußbewegungen oder schnelles Laufen Probleme? 


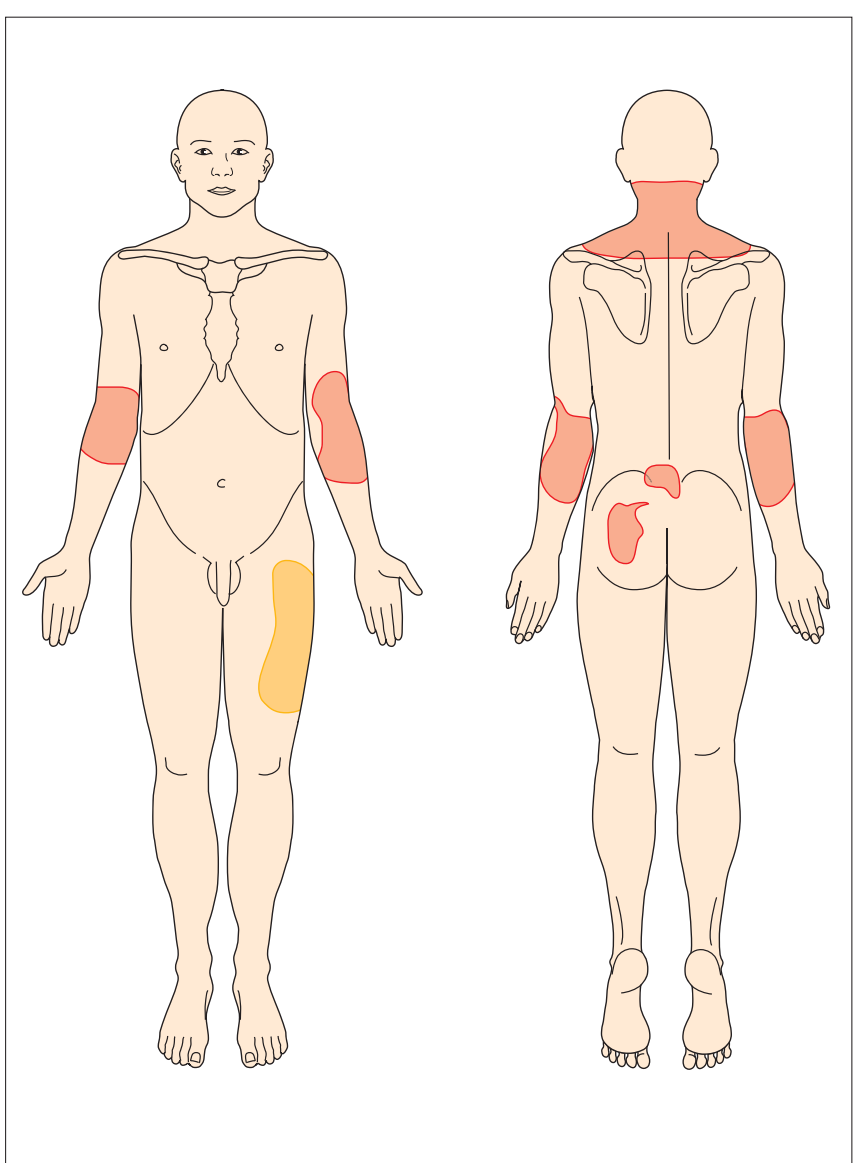

Abb. 1 Körpertabelle: Sabine hat Schmerzen in beiden Ellenbogengelenken und dem Schulter-Nacken-Bereich sowie ein brennendes Gefühl in der LWS. Die Haut an ihrem linken Oberschenkel fühlt sich pelzig an. Am meisten Sorgen bereiten Sabine ihre Ellenbogengelenke.

$>$ Waren Sie schon mal bei einem Neurologen, um eine Multiple Sklerose, Amyotrophe Lateralsklerose oder einen Diabetes abklären zu lassen?

$>$ Gibt es in der Familie irgendwelche neurologischen Krankheiten? Sabine betont ausdrücklich, dass sie bislang noch keine Anzeichen für neurologische Krankheiten hatte und immer sehr fit war. Nachdem sie als Urlaubsvertretung vor sechs Jahren eine sehr monotone Arbeit („schrauben und drehen“) durchgeführt hat, hatte sie zwar eine Sehnenscheidenentzündung, diese war aber nach zwei Wochen wieder weg.

\section{Untersuchung}

Momentan spürt Sabine ihre Nackenschmerzen (VAS 7/10) sowie die Schmerzen in beiden Ellenbogen (VAS 6/10).

\section{Inspektion der Nacken-Schulter-Region}

$>$ Schultern beidseits protrahiert. Durch Korrektur verändern sich die Beschwerden in den Armen und im Nacken nicht.

$>$ keine Atrophien in den Schulter-, Arm- und Handmuskeln

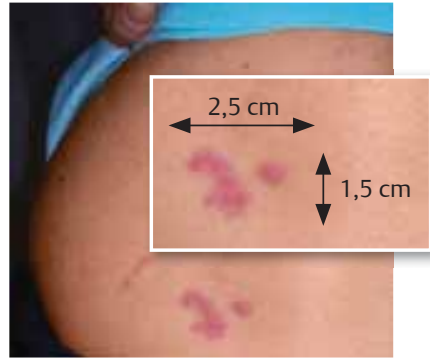

Abb. 2 Der rote Fleck: Die Hauterscheinung auf Sabines LWS misst etwa $2,5 \times 1,5 \mathrm{~cm}$. Konzentriert sich Sabine darauf, spürt sie ein leichtes Jucken und Brennen.

\section{Inspektion der LWS und des Beckens}

$>$ Haut: Paravertebral befindet sich links ein kleiner, roter, nicht schmerzhafter Fleck (॰ Abb. 2).

$>$ kein Shift, keine Positionsveränderungen des Beckens

$>$ keine Ausweichhaltungen

$>$ Muskelrelief o. B.

\section{Neurologische Untersuchung der oberen Extremitäten}

$>$ Kraft, Reflexe und Sensibilität o. B.

$>$ Diadochokinese 0 . B.

\section{Neurologische Untersuchung der unteren Extremitäten}

$>$ Kraft und Reflexe L2-S2 o. B.

> Sensibilität: wechselnde hyperalgetische Regionen am lateralen linken Oberschenkel und lateral des Knies. Bei oberflächlicher Pal pation hat Sabine das Gefühl, dass es sich pelzig anfühlt.

> Babinski-Reflex o. B.

\section{Neurodynamische Untersuchung}

$>$ ULNT 1 und 3 beidseits 0 . $B$.

> Passive Nackenflexion: „Ziehen“ im Nacken (VAS 4/10), ähnlich wie die Nackenbeschwerden. Verstärkt durch zervikalen SlumpTest (VAS 7/10) (๑ Abb. 3, S. 42). Nach der Extension im Kniegelenk links bekommt Sabine ein leichtes „Kribbeln“ und „Jucken“ im linken Gesäßbereich. Die Kniegelenkextension ist jedoch nicht eingeschränkt.

> Straight-Leg-Raise-Test: „Ziehen“ in der Kniekehle bis Kalkaneus bei $90^{\circ}$ Hüftgelenkflexion (re.: VAS 4/10; Ii.: VAS 6/10). Zwei Sekunden nachdem das linke Bein wieder in neutraler Position liegt, beginnt ein leichtes „Jucken“ und „Kribbeln“ im linken Gesäßbereich. Dies hält ungefähr 10-15 Sekunden lang an.

> Beim modifizierten Slump-Test mit Lateralflexion des Rumpfes nach rechts sowie Adduktion und Innenrotation im linken Hüftgelenk kommt das „Kribbeln“ sofort (॰ Abb. 4, S. 42). Nackenextension verändert das Kribbeln nicht.

\section{Physiologische Bewegungen der HWS mit Überdruck}

$>$ Extension, Lateralflexion und Rotation o. B.

$>$ bei Flexion „Ziehen“ im Nacken (VAS 5/10)

\section{Untersuchung des Ellenbogengelenks}

$>$ Flexion bds. sowie Pro- und Supination in $90^{\circ}$ Flexion o. B. 


\section{physiotherapie}

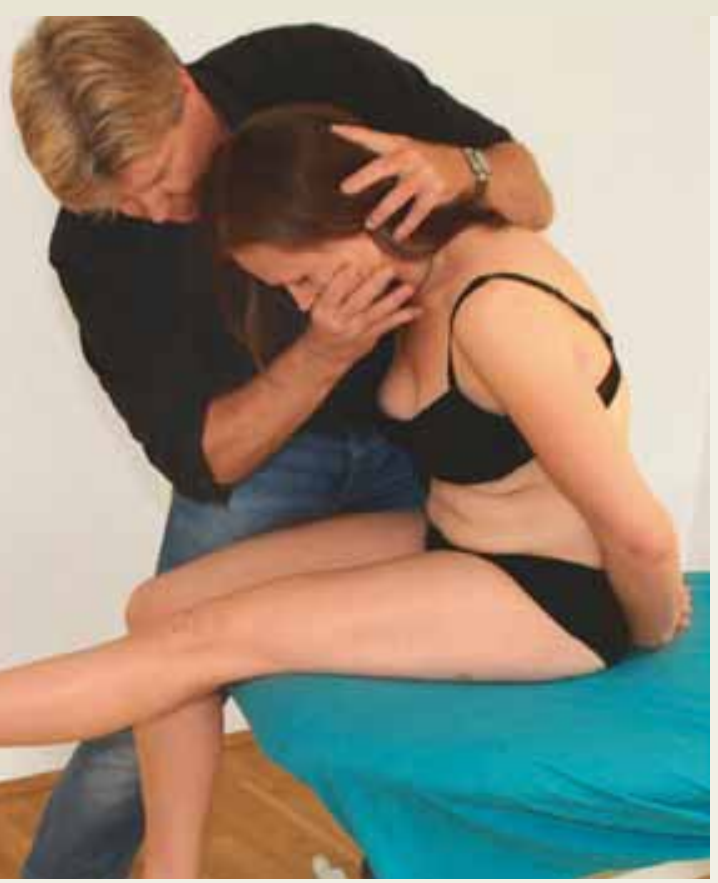

Abb. 3 Zervikaler Slump-Test: Nach der Kniegelenkextension bekommt Sabine ein leichtes „Kribbeln“ und "Jucken“ im linken Gesäßbereich.

$>$ Extension sowie Abduktion und Adduktion bei extendiertem Ellenbogen: minimale Krepitation bei $180^{\circ}$ und lokaler Schmerz (li.: VAS 4/10; re.: VAS 6/10)

\section{Physiologische Bewegungen der LWS mit Überdruck}

$>$ aktive Extension, Lateralflexion und Rotation o. B.

$>$ bei Flexion leichtes „Ziehen“ im linken Gesäßbereich

Es lassen sich weder deutliche neurologische und "klassische“ neurodynamische Symptome in der oberen Extremität feststellen noch ein klares neuromuskuloskeletales Muster. Nur die endgradigen Tests für das Ellenbogengelenk sind stark sensitiv. Auffällig ist, dass ich die Beschwerden teilweise bei endgradigen Gelenktests und teils bei neurodynamischen Tests reproduzieren kann, ohne dass ich einen abnormen Widerstand spüre. Möglicherweise handelt es sich um eine sekundäre Hyperalgesie, die nicht durch das neuromuskuloskeletale System verursacht wird. Etwas merkwürdig finde ich den roten Fleck auf Sabines Rücken. Er lässt mich an Hauterkrankungen denken - beispielsweise den Herpes Zoster - , die bei Patienten unter anderem zu "muskuloskeletalen“ Beschwerden führen können. Da der Fleck momentan das ist, was ich mit am auffälligsten finde, stelle ich noch einige Fragen dazu.

\section{Fragen bezüglich des roten Flecks auf Sabines Rücken}

$>$ Hatten Sie schon einmal solch einen Fleck irgendwo am Körper? $>$ Juckt der Fleck?

$>$ Hatten Sie im Kindesalter alle Kinderkrankheiten?

$>$ Sind bei Ihnen Hautkrankheiten wie Skelerodermie oder Neurodermitis bekannt?

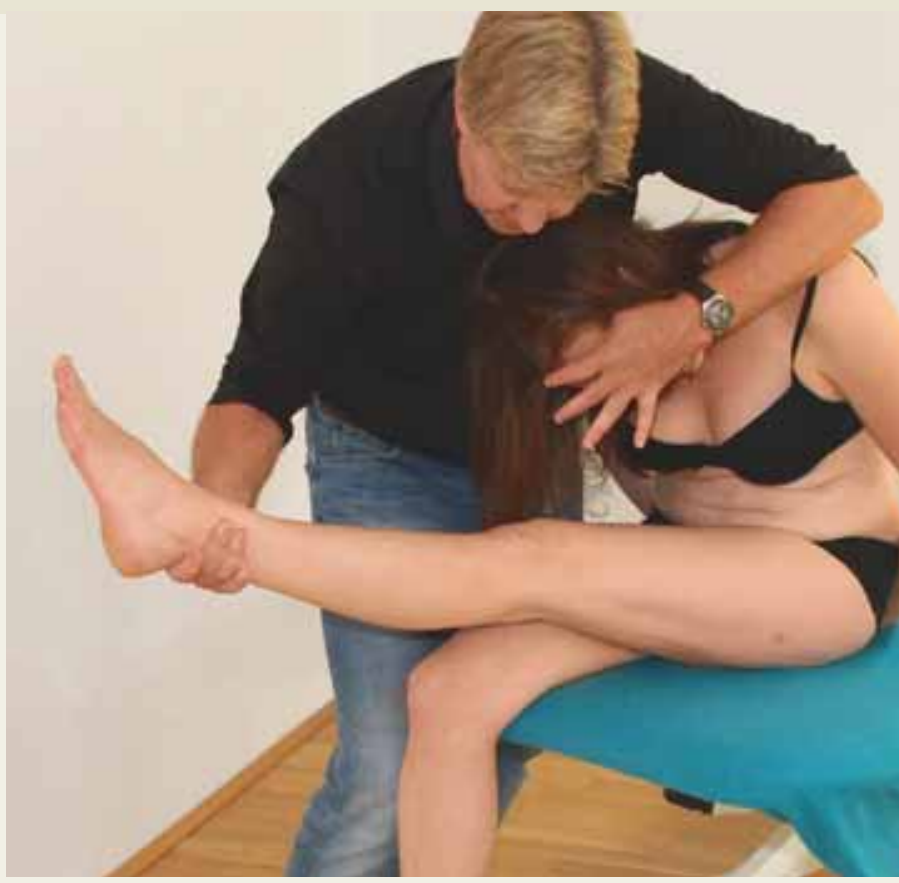

Abb. 4 Modifizierter Slump-Test: Um die Symptome weiter zu differenzieren, ergänzt Harry von Piekartz den Test mit Lateralflexion des Rumpfes nach rechts und Hüftgelenkadduktion des linken Beines.

> Hatten Sie schon einmal oder haben Sie momentan irgendwelche allergischen Reaktionen?

Sabine hat den roten Fleck auf ihrem Rücken bisher nicht bemerkt. Auch sonst konnte sie an ihrem Körper keine ähnlichen Hautveränderungen beobachten. Wenn sie sich auf die Stelle des Flecks konzentriert, hat sie das Gefühl, dass er etwas brennt und juckt. Bei ihr sind keine Allergien oder Hautkrankheiten bekannt und sie hatte bereits alle Kinderkrankheiten - inklusive Windpocken. Um die Ursache für die Hautveränderungen abklären zu lassen, überweise ich Sabine mit einem Bericht zum Hautarzt.

So ging es weiter $>$ Zwei Tage nach unserem Termin schickte mir Sabine eine Nachricht und ein Foto ihres Rückens. Das brennende, juckende Gefühl hatte sich vermehrt, und auch die linke Gesäßhälfte war nun betroffen. Sie schrieb außerdem, dass die Missempfindungen in ihrem linken Bein unverändert waren, aber die Haut am Oberschenkel keine Verfärbungen aufzeigte. Am darauffolgenden Tag hatte sie den Termin beim Hautarzt.

Der Hautarzt diagnostizierte einen Herpes Zoster. Er riet Sabine, sich eine Woche zu schonen, und verschrieb ihr das Virostatikum Aciclovir, das beispielsweise in dem Medikament "Zovirax oral“ enthalten ist. Weiter sollte sie auf eine optimale Hygiene achten. Der Arzt machte sie zudem darauf aufmerksam, dass ihre Erkrankung für alle ansteckend ist, die bislang keine Windpocken oder einen Herpes Zoster hatten.

Nach sechs Tagen waren das brennende und juckende Gefühl im Rücken und die Missempfindungen in den Beinen deutlich ver- 
ringert sowie der Hautausschlag sichtbar zurückgegangen. Die Nackenschmerzen und die Schmerzen in den Ellenbogengelenken hatten sich um 50\%verringert. Nach 14 Tagen waren Sabines Beschwerden kaum noch vorhanden, nur die Haut an der Lendenwirbelsäule zeigte noch leichte Auffälligkeiten.

\section{Eine kurze Reflexion}

Die Gelenktests und die neurodynamischen Tests waren bei Sabine offenbar falsch positiv. Möglicherweise schwächten die erhöhte Belastung (Umzug, wenig Schlaf etc.) ihr Immunsystem, wodurch das Herpes-Zoster-Virus aktiviert wurde (๑ "Hintergrundwissen“). Die Folge könnte eine sekundäre Hyperalgesie gewesen sein.

Harry von Piekartz

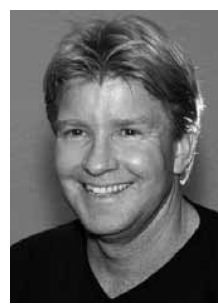

Prof. Dr. Harry von Piekartz ist Professor für Physiotherapie an der Hochschule Osnabrück, Präsident der Cranio Facial Therapy Academy (Crafta) sowie Fachlehrer für das Neuro-orthopädische Institut (NOI) und das Maitland-Konzept (IMTA). In der Artikelserie „Red Flags erkennen“ beschreibt er Fälle aus der eigenen Praxis.

\section{Herpes-Erkrankungen}

Eine Herpes-Zoster-Erkrankung geht immer auf eine vorausgegangene Infektion mit dem Varicella-Zoster-Virus (VZV) zurück. Dieses gehört zu den Viren der Herpes-Gruppe.

\section{Viren der Herpes-Gruppe (Herpesviridae)}

\begin{tabular}{|c|c|c|}
\hline Name & Symptome bei Infektion & Bemerkung \\
\hline $\begin{array}{l}\text { Herpes-simplex- } \\
\text { 1-Virus (HSV 1) }\end{array}$ & $\begin{array}{l}\text { Juckende, ansteckende Bläschen, die sich in Gruppen formieren, v. a. im } \\
\text { Gesicht (Lippen). Übertragung durch Speichelkontakt. Nach der Erstinfek- } \\
\text { tion persistieren die Viren in den Gliazellen der Spinalganglien und können } \\
\text { durch bestimmte Auslöser lebenslang rezidivieren, z. B. bei abgeschwäch- } \\
\text { ter Immunlage (Menstruation, intensive Sonnenbestrahlung), aber auch al- } \\
\text { lein durch Vorstellungskraft („Ekelbläschen“). }\end{array}$ & $\begin{array}{l}\text { Tritt überwiegend als Herpes labiales } \\
\text { (Lippenherpes) in Erscheinung; Prävalenz } \\
\text { in der deutschen Bevölkerung: ca. } 90 \% \text {. } \\
\text { Keine Impfung möglich. }\end{array}$ \\
\hline $\begin{array}{l}\text { Herpes-simplex- } \\
\text { 2-Virus (HSV 2) }\end{array}$ & $\begin{array}{l}\text { Schmerzhafte, juckende Bläschen im Genitalbereich. Übertragung durch } \\
\text { engen Schleimhautkontakt, z. B. beim Geschlechtsverkehr. Die Prävalenz } \\
\text { hängt stark von Alter und sexueller Promiskuität ab (häufige Verbreitung } \\
\text { in Verbindung mit anderen Geschlechtskrankheiten). }\end{array}$ & $\begin{array}{l}\text { Tritt überwiegend als Herpes genitalis } \\
\text { in Erscheinung. Bislang keine Impfung } \\
\text { möglich. }\end{array}$ \\
\hline $\begin{array}{l}\text { Varicella-Zoster- } \\
\text { Virus (VZV) }\end{array}$ & $\begin{array}{l}\text { Löst im Erstkontakt Windpocken (Varizellen) und bei Rezidiven die Gürtel- } \\
\text { rose (Herpes Zoster) aus. Eine Impfung ist möglich und wird seitens der } \\
\text { Ständigen Impfkommission empfohlen. }\end{array}$ & $\begin{array}{l}\text { Ansteckungsgefahr besteht nur für jemand, } \\
\text { der bisher keine Varizellen hatte. Menschen, } \\
\text { die schon Windpocken hatten, können sich } \\
\text { also nicht mit Herpes Zoster anstecken. }\end{array}$ \\
\hline $\begin{array}{l}\text { Ebstein-Barr-Virus } \\
\text { (EBV) }\end{array}$ & $\begin{array}{l}\text { Erreger des „Pfeifferschen Drüsenfiebers“; wegen seines Infektionsweges } \\
\text { auch als „Kusskrankheit“ bekannt und korrekter als „infektiöse Mononuk- } \\
\text { leose“ bezeichnet. Im Kindesalter verläuft es oft wie eine Grippe, im } \\
\text { Erwachsenenalter gibt es schwerere Verläufe mit Halsschmerzen, Lymph- } \\
\text { knotenschwellungen, Fieberschüben und körperlicher Schwäche. }\end{array}$ & $\begin{array}{l}\text { Das EBV gehört zu den Onkoviren, ist } \\
\text { also in der Lage, Krebs auszulösen } \\
\text { (Burkitt-Lymphom, Nasopharynx-Karzinome). } \\
\text { Effekt einer Impfung bisher nicht klinisch } \\
\text { nachgewiesen. }\end{array}$ \\
\hline $\begin{array}{l}\text { Cytomegalie-Virus } \\
\text { (CMV) }\end{array}$ & $\begin{array}{l}\text { Zu } 40 \% \text { erfolgt die Infektion pränatal. Sie ist meist symptomlos, kann aber } \\
\text { auch zu geistiger/körperlicher Behinderung führen. Erwachsene sind vor } \\
\text { allem gefährdet, wenn sie an einem abgeschwächten Immunsystem leiden } \\
\text { (nach Transplantation, bei HIV-Infektion oder durch eine Krebserkrankung). }\end{array}$ & $\begin{array}{l}\text { Bei erwachsenen immunsupprimierten } \\
\text { Patienten kann das CMV Pneumonie, Kolitis, } \\
\text { Enzephalitis und Hepatitis auslösen; } \\
\text { u. U. letaler Verlauf. Keine Impfung möglich. }\end{array}$ \\
\hline
\end{tabular}

Tab. Die aufgeführten Viren gehören, bedingt durch ihre Zellstruktur, zur selben Gruppe, rufen aber sehr unterschiedliche Erkrankungen hervor. 


\section{Herpes-Erkrankungen}

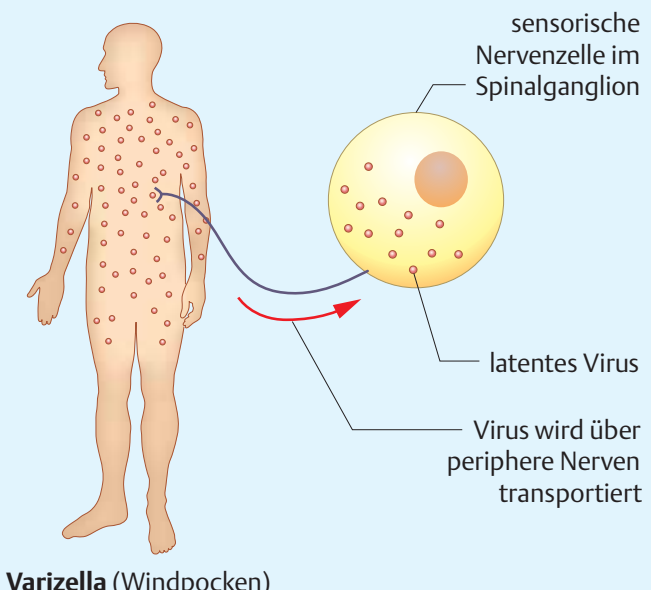

a Varizella (Windpocken)

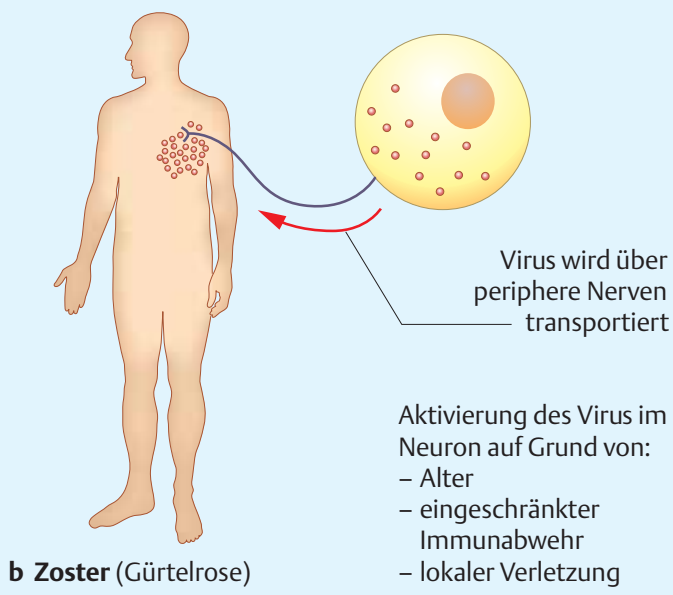

Abb. 5 Pathogenese des Herpes Zoster: Die Viren überleben in den Spinalganglien sensibler Hautnerven des Rückenmarks (a). Über bestimmte Faktoren kann das Virus im Neuron wieder aktiviert werden (b).

\section{Allgemeiner Infektionsweg}

Die Viren dringen beim ersten Kontakt in den Wirtsorganismus ein und infizieren bevorzugt den Übergang von Schleimhaut zu normaler Haut (Plattenepithelgewebe), um sich in den Epithelzellen zu vermehren. Denn Viren brauchen Wirtszellen für ihre Replikation. Dabei wird die Wirtszelle zerstört, was sich äußerlich durch die bläschenartige Entzündung zeigt und des Weiteren dafür sorgt, dass die Viren Kontakt zu den Endigungen sensibler Nerven finden und entlang des Axons (also retrograd) mit einer durchschnittlichen Geschwindigkeit von $0,7 \mu \mathrm{m} / \mathrm{s}$ zum Zellkörper des Neurons transportiert werden (๑ Abb. 5).

\section{Windpocken/Gürtelrose}

Das Varizella-Zoster-Virus kann, wie der Name schon verrät, zwei Krankheiten hervorrufen: primär Windpocken (Varizellen), sekundär den Herpes Zoster (Gürtelrose) (๑ Abb. 6). Windpocken sind eine typische Kinderkrankheit und haben einen hohen bundesdeutschen Durchseuchungsgrad (ca. 95\% der Erwachsenen).

Nach einer Primärinfektion mit dem VZV ist der Erkrankte, bedingt durch die Bildung von Antikörpern, lebenslang immun gegen VZV. Das bedeutet: Er kann zwar ein Rezidiv erleiden, sich aber nicht bei anderen Menschen anstecken. Durch Impfung mit Zostavax (empfohlen von der Ständigen Impfkommission des Robert-Koch-Institutes ab dem 1. Lebensjahr) werden solche Antikörper ebenfalls gebildet, ohne dass der Geimpfte die Krankheit selbst durchmachen muss.

Wie alle Viren der Herpes-Gruppe persistiert auch das VZV lebenslang, nachdem es einmal in den Organismus eingedrungen ist. Die Viren binden sich an sensible Nervenfasern und steigen entlang des Axons bis in die jeweiligen Spinalganglien oder, bei Hirnnervenbefall, in die Hirnnervenganglien, um dort (teils für Jahre bis Jahrzehnte) zu ruhen, bis eine entsprechende reduzierte Immunlage
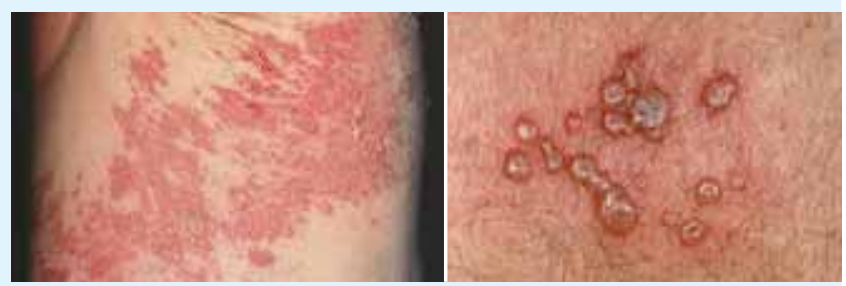

Abb. 6 Herpes Zoster: Die Erkrankung breitet sich meist dermatomal aus (li.). Typisch sind die kleinen Blässchen (re.).

die erneute innerindividuelle Ausbreitung erlaubt. Bei der Zweitund jeder weiteren Folgeerkrankung handelt es sich also immer um das Rezidiv einer vorherigen Windpockeninfektion.

Bei denjenigen Erwachsenen, die bisher weder die Krankheit durchgemacht haben, noch geimpft wurden, kommt es bei einer Primärinfektion zu schwereren Verläufen. Komplikationen bei Kindern und Erwachsenen sind unter anderem die VarizellenMeningitis und die Varizellen-Pneumonie.

$\rightarrow$ Informationen zum Impfschutz gibt es bei der Ständigen Impfkommission: www.rki.de > „Infektionsschutz" > „Impfen“.

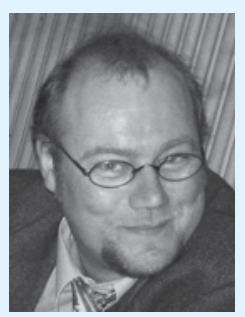

Prof. Dr. Christoff Zalpour ist Mediziner und Professor für Physiotherapie an der Hochschule Osnabrück. Dort leitet er das Institut für angewandte Physiotherapie und Osteopathie und ist Sprecher der AG Science in Physiotherapy in Osnabrück (SCIPOS). 